\title{
Reservoir Geophysics and Open Access Journal: Journal of Geophysics and Remote Sensing
}

\section{Rui Zhang*}

Institute for Geophysics, Jackson School of Geosciences, University of Texas at Austin

The reservoir geophysics is aimed at characterizationof reservoir by using geophysical data. Nowadays, the concept of reservoir geophysics becomes much broader than it was. On one side, it can provide more and more detailed subsurface structure and lithology information for geologists; on the other side, it can be utilized to capture fluid properties change after production. This shows a strong ability of reservoir geophysics as an effective way for connecting Geology and Reservoir Engineering. This also leads to a highly integration characteristics of reservoir geophysics, which needs calibration widely with both geologists and reservoir engineers.

Many issues are important for reservoir geophysics, and needed for more study. For example, rock physics model is one of the key points to link seismic data to rock properties, such as porosity and fluid saturation. Ultra-thin bed inversion is another interesting topic for reservoir geophysics to approach high resolution from conventional seismic data, because the detection for thin bed reservoir becomes more and more common during the development of old oil field. A new technique of micro-seismic method shows special ability for unconventional reservoir development by monitoring hydro fracture propagation. However, these studies are not independent and are highly integrated with other subjects. Thus, discussions become especially important for any successful applications. Journal of Geophysics and Remote Sensing (JGRS) emerges as new platform for communications and discussions.

Journal of Geophysics and Remote Sensing is an open access journal, which is a new style of publication. It provides a free platform for worldwide communication of technology. Through this platform, authors and readers can easily exchange their knowledge, experiences and interests. The free access to this new journal shows great advantage over most traditional journals for readers to get the latest progress in this field. Any programs can be tested by someone else, and any applications can be discussed among whole community. Journal of Geophysics and Remote Sensing is started by OMICS Publishing Group, as a very professional publisher especially for open access journals. OMICS Publishing Group provides variety website-translation journals for about 50 languages. Readers from different native languages can benefit from communication by using the flexibility and interactive nature of Journal of Geophysics \& Remote Sensing.
*Corresponding author: Rui Zhang, Institute for Geophysics, Jackson School of Geosciences, University of Texas at Austin, USA $\square$ Tel: 832-588-3156, E-mail: rzhang@mail.utexas.edu

Received August 02, 2012; Accepted August 04, 2012; Published August 09, 2012

Citation: Zhang R (2012) Reservoir Geophysics and Open Access Journal: Journal of Geophysics and Remote Sensing. J Geophys Remote Sensing 1:e104 doi:10.4172/2169-0049.1000e104

Copyright: (c) 2012 Zhang R. This is an open-access article distributed under the terms of the Creative Commons Attribution License, which permits unrestricted use, distribution, and reproduction in any medium, provided the original author and source are credited. 